\title{
Salvage of Infected Breast Implants
}

\author{
Joon Ho Song ${ }^{1}$, Young Seok Kim ${ }^{1}$, Bok Ki Jung ${ }^{1}$, Dong Won Lee ${ }^{2}$, Seung Yong Song ${ }^{2}$, Tai Suk Roh ${ }^{1}$, \\ Dae Hyun Lew ${ }^{2}$ \\ ${ }^{1}$ Department of Plastic and Reconstructive Surgery, Institute for Human Tissue Restoration, Gangnam Severance Hospital, Seoul; \\ ${ }^{2}$ Department of Plastic and Reconstructive Surgery, Institute for Human Tissue Restoration, Severance Hospital, Yonsei University College of \\ Medicine, Seoul, Korea
}

Background Implant-based breast reconstruction is being performed more frequently, and implants are associated with an increased risk of infection. We reviewed the clinical features of cases of implant infection and investigated the risk factors for breast device salvage failure. Methods We retrospectively analyzed 771 patients who underwent implant-based breast reconstruction between January 2010 and December 2016. Age, body mass index, chemotherapy history, radiation exposure, and smoking history were assessed as potential risk factors for postoperative infection. We also evaluated the presence and onset of infection symptoms, wound culture pathogens, and other complications, including seroma, hematoma, and mastectomy skin necrosis. Additionally, we examined the mastectomy type, the use of acellular dermal matrix, the presence of an underlying disease such as hypertension or diabetes, and axillary node dissection.

Results The total infection rate was 4.99\% (58 of 1,163 cases) and the total salvage rate was $58.6 \%$ (34 of 58). The postoperative duration to closed suction drain removal was significantly different between the cellulitis and implant removal groups. Staphylococcus aureus infection was most frequently found, with methicillin resistance in $37.5 \%$ of the cases of explantation. Explantation after infection was performed more often in patients who had undergone 2-stage expander/implant reconstruction than in those who had undergone direct-to-implant reconstruction.

Conclusions Preventing infection is essential in implant-based breast reconstruction. The high salvage rate argues against early implant removal. However, when infection is due to methicillin-resistant $S$. aureus and the patient's clinical symptoms do not improve, surgeons should consider implant removal.

Keywords Breast implants / Infection / Methicillin-resistant Staphylococcus aureus / Seroma
Correspondence: Tai Suk Roh Department of Plastic and Reconstructive Surgery, Yonsei University College of Medicine, 211 Eonju-ro, Gangnam-gu, Seoul 06273, Korea

Tel: +82-2-2019-3422

Fax: +82-2-2019-3422

E-mail:ROHTS@yuhs.ac

\section{INTRODUCTION}

The frequency of post-mastectomy breast reconstruction is increasing, due to its benefits for body image, self-esteem, and quality of life. In the United States, breast reconstruction is the fifth most common reconstructive procedure performed by plastic surgeons [1]. Of the various breast reconstruction options, implant-based reconstruction is a relatively simple technique and has a shorter operative time than reconstruction with autologous tissue. Implant based reconstruction also has several 
other advantages, including the absence of donor-site morbidity, rapid patient recovery, and a shorter hospital stay [2].

The use of acellular dermal matrix (ADM) has been established as beneficial for prosthetic breast reconstruction. ADM provides support to mastectomy skin flaps, and minimizes fibrosis and the inflammatory response associated with the implant, thereby reducing capsular contracture [3].

Given these benefits, the use of implant-based breast reconstruction has increased in recent years. However, the incidence of implant infection following breast reconstruction after mastectomy has been reported to range from $2.5 \%$ to $24 \%$ [ 4 ]. In particular, infection requiring explantation remains the most devastating complication associated with implant-based breast reconstruction. Many treatment algorithms are available for preventing reconstructive failure and infection. We reviewed the rates of infection (defined as cellulitis) and implant removal at our institution. Characterizing the clinical differences between the patients who experienced cellulitis and those who underwent implant removal will provide knowledge that may be useful for improving the salvage rate after infection.

The use of implants is linked to an increased risk of infection. Many surgeons have described the risk factors for infection in patients undergoing implant-based breast reconstruction. However, few studies have compared the occurrence of cellulitis and the need for implant removal. Therefore, the purpose of this investigation was to review cases of postoperative implant infection and to identify the risk factors for failure to salvage the breast device.

\section{METHODS}

\section{Methods}

From January 2010 to December 2016, 5 plastic surgeons at our institution performed 1,163 cases of implant-based reconstruction in 771 patients after total mastectomy for breast cancer. We retrospectively reviewed the records of these patients.

The type of mastectomy and reconstruction performed by the surgical oncologist or plastic surgeon varied within our population. The mastectomy procedure was either a simple mastectomy or a modified radical mastectomy, depending on the results of the sentinel lymph node biopsy. All implants and expanders were placed in the subpectoralis muscle or using $\mathrm{ADM}$ as a sling. Interrupted 2-0 Vicryl sutures were used to affix the ADM, moving from the inframammary fold along the inferior breast. Drain insertion was defined as the placement of 1 or 2 drains per expander/implant. Drains were removed according to the amount and color of drainage.

Once every 2 weeks, in-office expansion was performed in tis- sue expander patients. Percutaneous injections of $50-100 \mathrm{~mL}$ of saline at a time were performed. The timing of expansion could be delayed depending on patients' postoperative treatment, such as chemotherapy or radiotherapy.

We defined cellulitis based on the criteria published by the Centers for Disease Control and Prevention, previously used by Ranganathan et al. [5], as follows: (i) a positive aseptically obtained culture, (ii) symptoms including whole breast erythema and swelling, or (iii) a negative aseptically obtained culture but a physician's diagnosis of infection for which antibiotics were prescribed [6]. Total reconstructive failure was defined as the requirement for complete explantation of the breast prosthesis.

For each patient, information about demographics and postoperative complications, including infection, was collected and analyzed. Demographic factors, including the patient's age, body mass index, history of chemotherapy, exposure to radiotherapy, and active smoking status, were assessed as possible risk factors for postoperative infection. In addition, the clinical features of patients who underwent explantation due to postoperative infection were analyzed. Clinical data were also investigated, including the presence and onset of infection symptoms and pathogens observed in cultures obtained from collected fluid or dehiscent wounds. Other postoperative complications, including seroma, hematoma, and mastectomy skin necrosis, were reviewed. Finally, mastectomy type and the use of $\mathrm{ADM}$ were also analyzed.

The chi-square and Fisher exact tests were used to evaluate categorical data, while the 2-sample t-test was used to evaluate continuous variables. We reported adjusted odds ratios (ORs), 95\% confidence intervals, and corresponding P-values based on the model. All statistical analyses were performed with SAS ver. 9.4 (SAS Institute, Inc., Cary, NC, USA), and statistical significance was set at $\mathrm{P}<0.05$.

\section{Management}

Implant-related infections were suspected in patients who experienced pain and erythema at the site of implantation in the presence of fever. Occasionally, ultrasonography was performed to visualize the periprosthetic fluid in order to identify the extent and location of the infection and its relationship with the implant. In patients with a large fluid collection, ultrasoundguided drainage was performed.

In patients suspected to have an implant-based infection, we carried out bacterial cultures from the surgical site or drained fluid, as well as blood tests, to identify the systemic infectious condition. Before culturing and checking for susceptibility to antibiotics, empiric broad-spectrum antibiotic therapy $(400 \mathrm{mg}$ of intravenous teicoplanin) was started. After the pathogen was 
identified, and its susceptibility to antibiotics was confirmed, the target therapy was started to eradicate the infection. If the patient's condition worsened or did not improve with adequate antibiotic therapy, we considered removing the implant. Patients with a systemic infection and poor general condition usually required immediate implant removal.

During the operation for implant removal, capsulectomy and debridement of the infected tissue were performed. The removed implant and tissue were analyzed for the presence of aerobic bacteria, anaerobic bacteria, fungi, and nontuberculous mycobacteria.

After implant removal, systemic antibiotics were administered for about 14 days, followed by oral antibiotics for 7 days. Re-implantation was recommended to patients 6-12 months later.

\section{Table 1. Characteristics of the patients}

\begin{tabular}{|lcc|}
\hline Characteristics & Infections (\%) & Total cases \\
\hline Total number & $58(4.99)$ & 1,163 \\
Direct to implant & $26(11.4)$ & 229 \\
Tissue expander & $32(3.43)$ & 934 \\
Median age (yr) & $45.6(29-69)$ & $45.2(18-83)$ \\
Body mass index $\left(\mathrm{kg} / \mathrm{m}^{2}\right)$ & $22.4(18.2-29.1)$ & $23.4(15.9-49.4)$ \\
\hline
\end{tabular}

\section{RESULTS}

\section{Patient characteristics}

From January 2010 through December 2016, implants were placed in 771 patients for reconstruction after total mastectomy. The total number of reconstructions was 1,163, and the number of treated breasts was 832 .

The characteristics of the patients who experienced a postoperative implant infection are shown in Table 1 . The infection rate was $4.99 \%$ (58 of 1,163 reconstructions). The median patient age was 45.6 years (range, 29-69 years), the median body mass index was $22.4 \mathrm{~kg} / \mathrm{m}^{2}$ (range, $\left.18.2-29.1 \mathrm{~kg} / \mathrm{m}^{2}\right)$, and the median follow-up period was 46 months (range, 16-65 months).

The demographic characteristics of the patients who had cellulitis and underwent explantation are shown in Tables 2 and 3. The total salvage rate was $58.6 \%$ ( 34 of the 58 cases of infection) (Table 4). There was a significant difference in the time to closed suction drain removal (postoperative days) between the cellulitis group and the implant removal group. A longer time went by before the closed suction drain was removed in the implant removal group; this may have been related to seroma, which was the most common complication of implant infection.

\section{Table 2. Details of cases of explantation}

\begin{tabular}{|c|c|c|c|c|c|c|c|c|c|c|}
\hline No. & $\begin{array}{l}\text { Age } \\
\text { (yr) }\end{array}$ & Smoking & HTN & DM & $\begin{array}{c}\text { Neoadjuvant } \\
\text { CTx }\end{array}$ & $\begin{array}{l}\text { Adjuvant } \\
\text { CTx }\end{array}$ & Postop RTx & $\begin{array}{c}\text { Type of } \\
\text { mastectomy }\end{array}$ & $\begin{array}{l}\text { Axillary node } \\
\text { dissection }\end{array}$ & ADM use \\
\hline 1 & 43 & No & No & No & No & Yes & No & TM & SLNB & No \\
\hline 2 & 33 & No & No & No & No & Yes & No & TM & SLNB & No \\
\hline 3 & 57 & No & Yes & Yes & No & No & No & TM & SLNB & No \\
\hline 4 & 39 & No & Yes & No & No & No & No & $\mathrm{TM}$ & ALND & No \\
\hline 5 & 40 & No & No & No & No & No & No & TM & SLNB & No \\
\hline 6 & 33 & No & No & No & No & Yes & Yes & $\mathrm{TM}$ & ALND & No \\
\hline 7 & 37 & No & No & No & No & No & No & TM & ALND & No \\
\hline 8 & 45 & Yes & No & No & No & Yes & No & TM & ALND & No \\
\hline 9 & 53 & No & No & No & No & No & No & TM & ALND & Yes \\
\hline 10 & 45 & No & Yes & No & Yes & No & Yes & TM & ALND & Yes \\
\hline 11 & 58 & No & Yes & No & No & No & No & TM & SLNB & No \\
\hline 12 & 47 & No & No & No & No & Yes & Yes & TM & ALND & Yes \\
\hline 13 & 47 & No & No & No & No & Yes & Yes & $\mathrm{TM}$ & ALND & Yes \\
\hline 14 & 51 & No & No & No & No & No & No & SSM & SLNB & Yes \\
\hline 15 & 45 & No & No & No & No & Yes & No & SSM & SLNB & Yes \\
\hline 16 & 38 & No & No & No & No & No & Yes & SSM & SLNB & Yes \\
\hline 17 & 45 & No & No & No & No & No & No & NSM & SLNB & Yes \\
\hline 18 & 50 & No & No & No & No & Yes & No & SSM & ALND & Yes \\
\hline 19 & 45 & Yes & No & No & No & Yes & No & NSM & ALND & Yes \\
\hline 20 & 50 & No & No & No & No & Yes & Yes & NSM & ALND & Yes \\
\hline 21 & 51 & No & No & No & No & Yes & No & SSM & SLNB & Yes \\
\hline 22 & 62 & No & No & No & Yes & Yes & Yes & NSM & ALND & Yes \\
\hline 23 & 46 & No & No & No & No & No & No & TM & SLNB & Yes \\
\hline 24 & 54 & No & No & No & No & Yes & No & SSM & ALND & Yes \\
\hline
\end{tabular}


Table 3 . Details of cases of cellulitis

\begin{tabular}{|c|c|c|c|c|c|c|c|c|c|c|}
\hline No. & $\begin{array}{l}\text { Age } \\
\text { (yr) }\end{array}$ & Smoking & HTN & DM & $\begin{array}{l}\text { Neoadjuvant } \\
\text { CTx }\end{array}$ & $\begin{array}{l}\text { Adjuvant } \\
\text { CTx }\end{array}$ & $\begin{array}{c}\text { Adjuvant } \\
\text { RTx }\end{array}$ & $\begin{array}{c}\text { Type of } \\
\text { mastectomy }\end{array}$ & $\begin{array}{l}\text { Axillary node } \\
\text { dissection }\end{array}$ & ADM use \\
\hline 1 & 35 & No & No & No & No & No & No & $\mathrm{TM}$ & SLNB & No \\
\hline 2 & 69 & No & Yes & No & No & No & No & NSM & SLNB & No \\
\hline 3 & 51 & No & No & No & No & No & No & NSM & ALND & No \\
\hline 4 & 49 & No & No & No & No & No & No & $\mathrm{TM}$ & ALND & No \\
\hline 5 & 33 & No & No & No & No & Yes & Yes & $\mathrm{TM}$ & SLNB & No \\
\hline 6 & 46 & No & Yes & Yes & No & Yes & No & $\mathrm{TM}$ & ALND & No \\
\hline 7 & 49 & No & Yes & No & No & No & No & $\mathrm{TM}$ & SLNB & No \\
\hline 8 & 42 & No & No & No & No & Yes & No & $\mathrm{TM}$ & ALND & Yes \\
\hline 9 & 52 & No & No & No & No & No & No & $\mathrm{TM}$ & SLNB & Yes \\
\hline 10 & 37 & No & No & No & Yes & No & Yes & $\mathrm{TM}$ & ALND & Yes \\
\hline 11 & 60 & No & No & Yes & No & Yes & Yes & $\mathrm{TM}$ & ALND & No \\
\hline 12 & 32 & No & No & No & Yes & No & Yes & $\mathrm{TM}$ & ALND & No \\
\hline 13 & 62 & No & No & No & No & No & No & SSM & SLNB & Yes \\
\hline 14 & 43 & No & No & No & Yes & No & No & $\mathrm{TM}$ & SLNB & Yes \\
\hline 15 & 35 & No & No & No & No & No & No & NSM & SLNB & Yes \\
\hline 16 & 38 & No & No & No & No & Yes & Yes & NSM & ALND & Yes \\
\hline 17 & 58 & No & No & No & No & Yes & No & $\mathrm{TM}$ & ALND & Yes \\
\hline 18 & 45 & No & No & No & No & No & No & NSM & ALND & Yes \\
\hline 19 & 29 & No & No & No & Yes & No & Yes & NSM & SLNB & Yes \\
\hline 20 & 29 & No & No & No & Yes & No & Yes & NSM & ALND & Yes \\
\hline 21 & 38 & No & No & No & No & Yes & No & NSM & ALND & Yes \\
\hline 22 & 29 & No & No & No & Yes & No & Yes & NSM & ALND & Yes \\
\hline 23 & 41 & No & No & No & No & No & No & NSM & SLNB & Yes \\
\hline 24 & 53 & No & Yes & No & No & Yes & No & NSM & SLNB & Yes \\
\hline 25 & 56 & No & No & No & No & Yes & No & NSM & ALND & Yes \\
\hline 26 & 52 & Yes & No & Yes & No & Yes & No & SSM & SLNB & Yes \\
\hline 27 & 48 & No & No & No & No & Yes & No & NSM & ALND & Yes \\
\hline 28 & 35 & No & No & No & No & No & No & NSM & SLNB & Yes \\
\hline 29 & 55 & No & No & No & No & Yes & No & NSM & SLNB & Yes \\
\hline 30 & 44 & No & Yes & No & No & No & No & NSM & SLNB & Yes \\
\hline 31 & 44 & No & No & No & No & No & No & SSM & SLNB & Yes \\
\hline 32 & 47 & No & No & No & Yes & No & Yes & NSM & ALND & Yes \\
\hline 33 & 42 & No & No & No & No & No & No & NSM & SLNB & Yes \\
\hline 34 & 50 & No & No & No & No & No & No & NSM & SLNB & Yes \\
\hline
\end{tabular}

Explantation after breast implant infection was performed more frequently in patients who underwent 2-stage expander/implant reconstruction than in those who underwent direct-to-implant reconstruction. A univariate logistic regression analysis found that 2-stage breast reconstruction was associated with 5.5 times higher odds of explantation after breast implant infection than 1-stage breast reconstruction (Table 5).

\section{Bacterial culture pathogens}

The microorganism most frequently observed in our study was Staphylococcus aureus, with methicillin resistance observed in $21.4 \%$ of the cases of infection. Acinetobacter baumannii was found in $14.3 \%$ of patients, and Staphylococcus epidermidis was reported in $8.93 \%$. Of note, no bacterial growth was observed in $25.0 \%$ of patients with a postoperative infection (Table 6).
A binary logistic regression analysis identified 2 bacterial infections that were predictors of implant removal: methicillin-resistant S. aureus (MRSA) (OR=15.743; 95\% CI, 2.398-103.357) and $A$. baumannii $(\mathrm{OR}=9.114 ; 95 \%$ confidence interval $[\mathrm{CI}]$, 1.254-66.25) (Table 7).

\section{Related wound complications}

Other wound complications occurred, including seroma, wound dehiscence, skin flap necrosis, capsular contracture, and hematoma (Table 8). Seroma was noted in 23 of 58 patients. Skin flap necrosis was noted in 5 patients and hematoma occurred in five patients. Five patients with skin flap necrosis underwent revision surgery. Capsular contracture was noted in 2 patients, both of whom underwent capsulectomy during explantation. 
Table 4. Procedural and clinical characteristics of patients who experienced cellulitis or reconstruction failure

\begin{tabular}{|lccc|}
\hline Characteristic & Cellulitis & Implant removal & P-value \\
\hline Total number & $34(58.6)$ & $24(41.4)$ & - \\
Direct to implant & $22(78.6)$ & $6(21.4)$ & 0.0029 \\
Tissue expander & $12(40.0)$ & $18(60.0)$ & \\
Median age (yr) & $44.9(34-69)$ & $46.4(18-83)$ & 0.5451 \\
Body mass index (kg/m²) & $22.0(19.2-29.1)$ & $21.4(18.2-25.9)$ & 0.4027 \\
Onset time of infection & $20(5-150)$ & $16.5(3-191)$ & 0.3201 \\
Neoadjuvant CTx & $7(20.59)$ & $2(8.33)$ & 0.2816 \\
Adjuvant CTx & $12(35.29)$ & $13(54.17)$ & 0.1529 \\
Adjuvant RTx & $9(26.47)$ & $7(29.17)$ & 0.8210 \\
HTN & $5(14.71)$ & $4(16.67)$ & 0.9999 \\
DM & $3(8.82)$ & $1(4.17)$ & 0.6351 \\
Smoking Hx & $1(2.94)$ & $2(8.33)$ & 0.5637 \\
ADM use & $25(73.53)$ & $15(62.5)$ & 0.3712 \\
Axillary node dissection & $16(47.06)$ & $13(54.17)$ & 0.5939 \\
HV removal date \#1 (POD) & $11(3-40)$ & $18(5-38)$ & 0.0433 \\
HV removal date \#2 (POD) & 16.5 (3-52) & $20(5-29)$ & 0.8642 \\
\hline Values are presented as median (range) or number (\%). & \\
CTx, chemotherapy; RTx, radiotherapy; HTN, hypertension; DM, diabetes mellitus; & \\
Hx, history; ADM, acellular dermal matrix; HV, Hemovac; POD, postoperative day. \\
\hline
\end{tabular}

Table 5. Univariate logistic regression for implant removal

\begin{tabular}{|lcc|}
\hline Variable & $\begin{array}{c}\text { Odds ratio (95\% } \\
\text { confidence interval) }\end{array}$ & P-value \\
\hline $\begin{array}{c}\text { Two-stage expander/ } \\
\text { implant reconstruction }\end{array}$ & $5.5(1.722,17.566)$ & 0.004 \\
\hline
\end{tabular}

\section{Table 6. Pathogens in bacterial cultures}

\begin{tabular}{|lccc|}
\hline Bacterial culture & No. (\%) & Cellulitis & Explantation \\
\hline $\begin{array}{l}\text { Gram-positive } \\
\text { Methicillin-resistant } \\
\quad \text { Staphylococcus aureus }\end{array}$ & $12(21.4)$ & $3(9.38)$ & $9(37.5)$ \\
$\begin{array}{l}\text { Methicillin-sensitive } \\
\quad \text { Staphylococcus aureus }\end{array}$ & $7(12.5)$ & $6(18.75)$ & $1(4.17)$ \\
$\quad$ Staphylococcus epidermidis & $5(8.93)$ & $2(6.25)$ & $3(12.5)$ \\
$\quad$ Methicillin-resistant & $4(7.14)$ & $3(9.38)$ & $1(4.17)$ \\
$\quad$ Staphylococcus epidermidis & & & \\
$\quad$ Other & $3(5.36)$ & $2(6.25)$ & $1(4.17)$ \\
Gram-negative & & & \\
$\quad \begin{array}{l}\text { Acinetobacter baumannii } \\
\text { Pseudomonas aeruginosa }\end{array}$ & $8(14.3)$ & $3(9.38)$ & $5(20.83)$ \\
No growth & $3(5.36)$ & $1(3.13)$ & $2(8.33)$ \\
\hline
\end{tabular}

\section{DISCUSSION}

This retrospective investigation included a large series of implant-based reconstructions $(n=1,163)$ performed at a single medical center from January 2010 to December 2016.

Infectious complications of implant-based breast reconstructions are a significant cause of morbidity. Infectious complica-
Table 7. Univariate logistic regression for implant removal

\begin{tabular}{lcc}
\hline Variable & $\begin{array}{c}\text { Odds ratio (95\% } \\
\text { confidence interval) }\end{array}$ & P-value \\
\hline MRSA vs. no growth & $15.743(2.398,103.357)$ & 0.0041 \\
CNS vs. no growth & $8.12(0.853,77.257)$ & 0.0684 \\
Pseudomonas aeruginosa vs. no growth & $9.671(0.639,146.263)$ & 0.1016 \\
Acinetobacter baumannii vs. no growth & $9.114(1.254,66.25)$ & 0.0290 \\
Other pathogen vs. no growth & $1.765(0.276,11.304)$ & 0.5486 \\
\hline MRSA, methicillin-resistant Staphylococcus aureus; CNS, coagulase-negative \\
Staphylococcus. & & \\
\hline
\end{tabular}

Table 8. Wound complications in patients with a postoperative implant infection

\begin{tabular}{|lrcc|}
\hline Wound complication & No. & $\begin{array}{c}\text { Cellulitis } \\
(\%)\end{array}$ & Explantation \\
\hline Seroma & 23 & $15(65.2)$ & $8(34.8)$ \\
Wound dehiscence and skin flap necrosis & 5 & $3(60)$ & $2(40)$ \\
Hematoma & 5 & 0 & $5(100)$ \\
Capsular contracture (at least grade III) & 2 & 0 & $2(100)$ \\
No other complication & 31 & $19(57.6)$ & $14(42.4)$ \\
\hline
\end{tabular}

tions may result in hospital readmission, delayed chemotherapy and/or radiation therapy, and explantation. Our definition of cellulitis related to implant infection was based on the Centers for Disease Control and Prevention criteria, previously used by Ranganathan et al. [5]

In other studies, the definition of infection was either unclear or broader than the definition used in the present study (i.e., including patients treated with oral antibiotics at an outpatient clinic) $[4,7]$. Pinsolle et al. [7] reported an implant-associated infection rate of $13 \%$. In our study, the total infection rate for implant-based breast reconstructions was $4.99 \%$, and the salvage rate was $58.6 \%$. Once infection is detected, appropriate initial antibiotic therapy should be started as soon as possible. The $58.6 \%$ salvage rate with antibiotic therapy observed in this study is very acceptable, and argues against early implant removal $[8,9]$. However in a statistical comparison of cases identified as having MRSA, A. baumannii, and no culture growth, the OR for explantation in the MRSA and A. baumannii groups was meaningfully high. This observation suggests that implant removal can be considered when clinical signs worsen and/or when MRSA or A. baumannii is identified. Spear and Seruya [10] reported a high risk of reconstruction failure in infections caused by Gram-negative bacteria or MRSA. According to other studies, the most common cause of breast infection was coagulasenegative Staphylococcus (CNS) [11]. CNS is known to be part of the endogenous flora and is associated with the periareolar approach. In our study, most of the implant-related infections were caused by MRSA, which might reflect the severity of infec- 
tions requiring explantation. MRSA is becoming increasingly more prominent, not only as a pathogen causing nosocomial infections, but also as a community-acquired pathogen causing severe skin and soft tissue infections. To prevent CNS, it is important to minimize skin contamination. Nipple shields may be helpful as well.

At our institution, explantation after breast implant infection was performed more frequently in patients who underwent 2-stage expander/implant reconstruction than in those who underwent direct-to-implant reconstruction. This may have been because of a higher risk of developing an ascending infection from endogenous skin flora and traumatic events during the expander inflation procedure. The need for a second operation for the implant expander may also increase the risk of infection.

Interestingly, our study showed a $25.0 \%$ rate of negative cultures of samples obtained from aspirated fluid or the surface of deep wounds. This finding might have resulted from prior antibiotic treatment if a patient was treated at the first sign or symptom of postoperative infection. In these cases, the use of appropriate antibiotics for management is difficult, and infection control can be delayed. However, our results indicated that when no pathogen was detected, the breast device could be salvaged with a high probability of success (Table 6).

In the present study, seroma formation was observed in 50\% of patients who underwent explantation. In addition, complications related to seroma occurred in 23 cases (Table 8). Several methods are available to help prevent seroma formation. Quilting sutures and the intraoperative inflation of tissue expanders with injectable saline are used to reduce dead space. Additionally, restricted exercise and elevation of the arm ipsilateral to the affected breast can help minimize seroma formation.

After mastectomy for the management of breast cancer, the breast parenchyma is removed, which can result in relative ischemia of the skin flaps due to disruption of the blood supply. For these reasons, the $\mathrm{ADM}$ might not function as expected. Additionally, pressure from the implants and the thinner mastectomy skin flap are associated with wound complications, such as wound dehiscence, skin flap necrosis, and seroma formation. In such cases, wound healing can be delayed, and infection is more likely to occur.

This study has some limitations. First, the definition of implant infection was restricted to cellulitis and implant explantation. Our reported infection rate seems to be underestimated. Subclinical infection or red breast syndrome were not analyzed in this study. Second, 5 plastic surgeons who used different techniques for implant-based breast reconstruction performed the reconstructions. Variations in implant irrigation, pocket irrigation, draping and glove changes, and other specific sterility tech- niques may have contributed to the infection rates and potentially altered the results. However, our large volume of cases may offset this limitation. Third, this study is limited by its retrospective nature. Fourth, we were not able to identify microorganisms in all cases of infection. In 2 cellulitis cases, we were not able to perform a bacteria culture. Lastly, the range of the $95 \%$ CIs for the risk of explantation after breast implant infection was large, due to the relatively small sample size.

The high salvage rate found in this study argues against early implant removal $[8,9]$. However, if a breast implant infection is associated with MRSA or A. baumannii and if the patient's clinical symptoms do not improve, surgeons should consider implant removal. Finally, seroma collection was the most common infection-related complication, and leading to drain prolongation and increasing the explantation rate. The results of this study will improve our understanding of the risk factors for infection and may help prevent infections in clinical practice.

\section{CONFLICT OF INTEREST}

No potential conflict of interest relevant to this article was reported.

\section{REFERENCES}

1. Weichman KE, Levine SM, Wilson SC, et al. Antibiotic selection for the treatment of infectious complications of implant-based breast reconstruction. Ann Plast Surg 2013;71: 140-3.

2. Cordeiro PG, McCarthy CM. A single surgeon's 12-year experience with tissue expander/implant breast reconstruction: part I. A prospective analysis of early complications. Plast Reconstr Surg 2006;118:825-31.

3. Nahabedian MY. Acellular dermal matrices in primary breast reconstruction: principles, concepts, and indications. Plast Reconstr Surg 2012;130(5 Suppl 2):44S-53S.

4. Francis SH, Ruberg RL, Stevenson KB, et al. Independent risk factors for infection in tissue expander breast reconstruction. Plast Reconstr Surg 2009; 124:1790-6.

5. Ranganathan K, Santosa KB, Lyons DA, et al. Use of acellular dermal matrix in postmastectomy breast reconstruction: are all acellular dermal matrices created equal? Plast Reconstr Surg 2015;136:647-53.

6. Pittman TA, Fan KL, Knapp A, et al. Comparison of different acellular dermal matrices in breast reconstruction: the 50/50 study. Plast Reconstr Surg 2017;139:521-8.

7. Pinsolle V, Grinfeder C, Mathoulin-Pelissier S, et al. Complications analysis of 266 immediate breast reconstructions. 
J Plast Reconstr Aesthet Surg 2006;59:1017-24.

8. Snyderman RK. Breast augmentation. In: Snyderman RK, editor. Symposium on Neoplastic and Reconstructive Problems of the Female Breast. St Louis: Mosby; 1973. p.32-7.

9. Southwick HW, Economou SG, Otten JW. Prosthetic replacement of chest-wall defects; an experimental and clinical study. AMA Arch Surg 1956;72:901-7.
10. Spear SL, Seruya M. Management of the infected or exposed breast prosthesis: a single surgeon's 15-year experience with 69 patients. Plast Reconstr Surg 2010;125:1074-84.

11. Nahabedian MY, Tsangaris T, Momen B, et al. Infectious complications following breast reconstruction with expanders and implants. Plast Reconstr Surg 2003;112:467-76. 